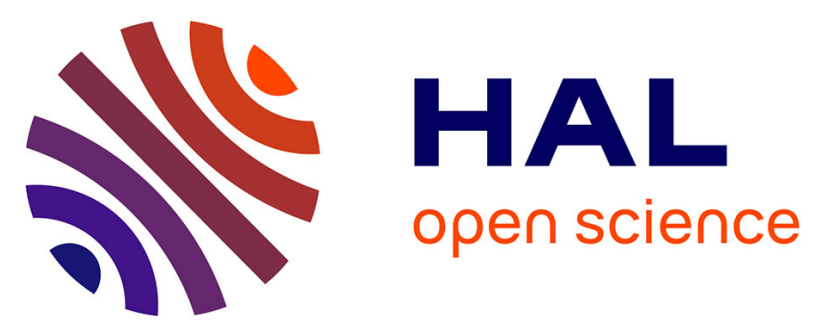

\title{
Variational Approach to Dynamic Brittle Fracture via Gradient Damage Models
}

\author{
Tianyi Li, Jean-Jacques Marigo, Daniel Guilbaud, Serguei Potapov
}

\section{To cite this version:}

Tianyi Li, Jean-Jacques Marigo, Daniel Guilbaud, Serguei Potapov. Variational Approach to Dynamic Brittle Fracture via Gradient Damage Models. Applied Mechanics and Materials, 2015, Damage Mechanics: Theory, Computation and Practice, 784, pp.334-341. 10.4028/www.scientific.net/AMM.784.334 . hal-01225237v2

\section{HAL Id: hal-01225237}

\section{https://hal.science/hal-01225237v2}

Submitted on 29 Dec 2015

HAL is a multi-disciplinary open access archive for the deposit and dissemination of scientific research documents, whether they are published or not. The documents may come from teaching and research institutions in France or abroad, or from public or private research centers.
L'archive ouverte pluridisciplinaire HAL, est destinée au dépôt et à la diffusion de documents scientifiques de niveau recherche, publiés ou non, émanant des établissements d'enseignement et de recherche français ou étrangers, des laboratoires publics ou privés. 


\title{
Variational Approach to Dynamic Brittle Fracture via Gradient Damage Models
}

\author{
Tianyi $\mathrm{Li}^{1, \mathrm{a}}$, Jean-Jacques Marigo ${ }^{2, \mathrm{~b}}$, Daniel Guilbaud ${ }^{1,3, \mathrm{c}}$, Serguei Potapov ${ }^{1,4, \mathrm{~d}}$ \\ ${ }^{1}$ Institut des Sciences de la Mécanique et Applications Industrielles, 92141 Clamart Cedex, France \\ ${ }^{2}$ Laboratoire de Mécanique des Solides, École Polytechnique, 91128 Palaiseau Cedex, France \\ ${ }^{3}$ CEA Saclay, 91191 Gif-sur-Yvette Cedex, France \\ ${ }^{4}$ EDF Lab, 92141 Clamart Cedex, France \\ atianyi.li@polytechnique.edu, bmarigo@Ims.polytechnique.fr \\ cdaniel.guilbaud@cea.fr, dserguei.potapov@edf.fr
}

Keywords: Dynamic fracture, Gradient damage models, Variational principles, Finite element implementation

\begin{abstract}
In this paper we present a family of gradient-enhanced continuum damage models which can be viewed as a regularization of the variational approach to fracture capable of predicting in a unified framework the onset and space-time dynamic propagation (growth, kinking, branching, arrest) of complex cracks in quasi-brittle materials under severe dynamic loading. The dynamic evolution problem for a general class of such damage models is formulated as a variational inequality involving the action integral of a generalized Lagrangian and its physical interpretation is given. Finite-element based implementation is then detailed and mathematical optimization methods are directly used at the structural scale exploiting fully the variational nature of the formulation. Finally, the link with the classical dynamic Griffith theory and with the original quasi-static model as well as various dynamic fracture phenomena are illustrated by representative numerical examples in quantitative accordance with theoretical or experimental results.
\end{abstract}

\section{Introduction}

Gradient damage models as formulated in a pure variational setting [1] provide, through strain and damage localization in narrow bands representing a regularized description of cracks, a complete and unified framework of brittle fracture including the onset and the space-time quasi-static propagation of cracks with possible complex topologies, see [2,3] and references therein. The presence of the damage gradient confirms the non-local nature of the model and induces naturally by dimensional analysis a material internal length. From the damage mechanics point of view, local damage models are mathematically ill-posed where damage localization is possible without any additional energy dissipation resulting in a spurious mesh dependence of the FEM results [4, 5]. The introduction of the damage gradient can thus be seen as a regularization of the classical continuum damage models to overcome this difficulty although other techniques are also available [6]. The link between damage and fracture can be established on one hand through $\Gamma$-convergence theories in terms of global minima of the total energy as long as this internal length is small before the size of the structure [7]. On the other hand, it is shown in [8] using matched asymptotic analysis, that the damage evolution ruled a priori by three physical principles of irreversibility, local directional stability and energy balance satisfies apparently the classical Griffith criterion through the definition of a fictitious energy release rate $\bar{G}$ of the outer problem and a material toughness $\overline{G_{\mathrm{c}}}$ proportional to the local damage dissipation and the internal length. This gradient damage model has been successfully applied to investigate among others thermal shocks $[9,10]$ and thin films debonding problems $[11,12]$.

We discuss in this work a natural dynamic extension of the original quasi-static gradient damage models $[1,2]$ to account for dynamic fracture phenomena. In presence of rapid propagation of cracks the quasi-static assumption is a priori not valid and inertial effects should be considered during the 
analysis. The reasoning in [13] still applies in dynamics concerning the inability of the classical Griffith theory of dynamic fracture mechanics to nucleate a crack in structures lacking sufficient initial singularities and to predict itself solely the crack path including kinking and branching without additional hypothesis such as the principle of local symmetry. As in the quasi-static setting, these issues can be directly addressed by an energy minimality principle in the dynamic gradient damage models. Dynamic fracture has already been studied using the so-called phase-field models $[14,15,16]$ which turn out to belong in fact to our general dynamic gradient damage models after a particular choice of damage constitutive laws. The other aim of this paper is to re-establish a certain link between damage and fracture in the dynamic setting through numerical examples and study convergence of the dynamic model towards the original quasi-static one with a vanishing loading speed in several circumstances.

This paper is organized as follows. The variational formulation of the dynamic gradient damage model is presented which constitutes a natural extension of the original quasi-static model. Numerical considerations are then discussed concerning in particular the temporal discretization of the time evolution problem. Finally, several representative numerical examples are given to provide some insights of the proposed formulation with respect to its use to approximate dynamic brittle fracture.

\section{Variational formulation of the dynamic gradient damage models}

We refer the reader to [1] and references therein for the basic variational ingredients and complete construction of gradient damage models. The first step is to introduce a new scalar damage field $0 \leq \alpha \leq 1$ depicting a continuous transition between the undamaged part $\alpha=0$ and the crack $\alpha=1$, see Fig. 1 .
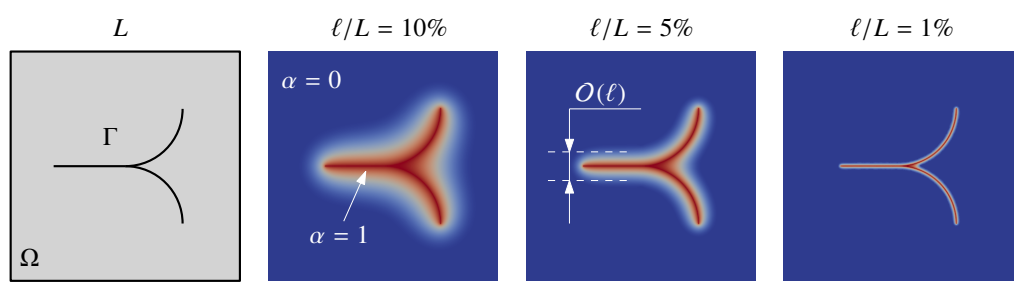

Figure 1: The discrete crack $\Gamma \subset \Omega$ approximated by a continuous damage field $0 \leq \alpha \leq 1$.

In the quasi-static model the principle of directional stability is physically feasible due to the minimization nature of the static equilibrium. But in dynamics we merely have a stationary Lagrangian so our approach here will be extending the first-order stability criterion. For formulational simplicities, we confine ourselves to the infinitesimal or linearized strain theory knowing that finite strain extension is also possible [17]. We reintroduce two basic energetic quantities used in quasi-static calculations: the elastic energy $\mathcal{E}\left(\mathbf{u}_{t}, \alpha_{t}\right)$ and the damage dissipation energy $\mathcal{S}\left(\alpha_{t}\right)$ which corresponds to the fracture surface energy of the structure $\Omega$

$$
\mathcal{E}\left(\mathbf{u}_{t}, \alpha_{t}\right)=\frac{1}{2} \int_{\Omega} a\left(\alpha_{t}\right) \mathrm{A} \boldsymbol{\varepsilon}\left(\mathbf{u}_{t}\right) \cdot \boldsymbol{\varepsilon}\left(\mathbf{u}_{t}\right), \quad \mathcal{S}\left(\alpha_{t}\right)=\int_{\Omega} w\left(\alpha_{t}\right)+w(1) \ell^{2} \nabla \alpha_{t} \cdot \nabla \alpha_{t}
$$

with $A$ the elasticity tensor, $\ell$ the above-mentioned internal length controlling the damage band (see Fig. 1) and $a(\alpha)$ along with $w(\alpha)$ two constitutive laws for damage describing respectively degradation of stiffness with damage and local damage dissipation. We are now in a position to bring the kinetic energy $\mathcal{K}\left(\dot{\mathbf{u}}_{t}\right)$ into the picture

$$
\mathcal{K}\left(\dot{\mathbf{u}}_{t}\right)=\frac{1}{2} \int_{\Omega} \rho \dot{\mathbf{u}}_{t} \cdot \dot{\mathbf{u}}_{t}
$$


and define the action integral of a generalized Lagrangian, counterpart of the quasi-static total potential energy $\mathcal{P}(\mathbf{u}, \alpha)$

$$
\mathcal{A}(\mathbf{u}, \alpha)=\int_{I} \mathcal{L}_{t}\left(\mathbf{u}_{t}, \dot{\mathbf{u}}_{t}, \alpha_{t}\right) \mathrm{d} t=\int_{I} \mathcal{E}\left(\mathbf{u}_{t}, \alpha_{t}\right)+\mathcal{S}\left(\alpha_{t}\right)-\mathcal{K}\left(\dot{\mathbf{u}}_{t}\right)-\mathcal{W}_{t}\left(\mathbf{u}_{t}\right) \mathrm{d} t
$$

where $I \subset \mathbb{R}$ denotes a certain physical time interval of interest and $\mathcal{W}$ the linear functional grouping all external loads. The coupled two-field time-continuous problem can then be formulated by the following three physical principles.

1. Irreversibility: the damage $\alpha(\cdot, \mathbf{x})$ is non-decreasing to prevent crack healing.

2. First-order stability: the action integral variation is always positive with respect to arbitrary test displacement $\mathbf{v}$ in the kinematic admissible space $C(\mathbf{u})$ incorporating Dirichlet boundary conditions and arbitrary test damage $\beta$ restricted to the damage admissible space $\mathcal{D}(\alpha)$ taking into account the irreversibility condition

$$
\mathcal{A}^{\prime}(\mathbf{u}, \alpha)(\mathbf{v}-\mathbf{u}, \beta-\alpha) \geq 0 \text { for all } \mathbf{v} \in \mathcal{C}(\mathbf{u}) \text { and } \beta \in \mathcal{D}(\alpha)
$$

3. Energy balance: the rate of the total energy should be equal to the total external power

$$
\dot{\mathcal{E}}_{t}+\dot{\mathcal{K}}_{t}+\dot{\mathcal{S}}_{t}=\mathcal{W}\left(\dot{\mathbf{u}}_{t}\right)+\int_{\partial \Omega_{\mathrm{D}}} a\left(\alpha_{t}\right) \mathrm{A} \boldsymbol{\varepsilon}\left(\mathbf{u}_{t}\right) \mathbf{n} \cdot \dot{\mathbf{U}}_{t}
$$

Wisely choosing the test functions $\mathbf{v}$ and $\beta$ in the variational inequality (1) and exploiting the topological natures of the two admissible spaces, we obtain the pointwise wave equation and the crack minimality criterion at the structural scale

$$
\left\{\begin{array}{l}
\rho \ddot{\mathbf{u}}_{t}=\operatorname{div}\left(a\left(\alpha_{t}\right) \operatorname{A} \boldsymbol{\varepsilon}\left(\mathbf{u}_{t}\right)\right)+\mathbf{f}_{t} \\
\mathcal{E}\left(\mathbf{u}_{t}, \alpha_{t}\right)+\mathcal{S}\left(\alpha_{t}\right) \leq \mathcal{E}\left(\mathbf{u}_{t}, \beta\right)+\mathcal{S}(\beta) \text { for all } 1 \geq \beta \geq \alpha_{t} \geq 0 .
\end{array}\right.
$$

Although (2b) is formally the same as in the quasi-static case, here the displacement $\mathbf{u}_{t}$ follows the elastodynamic equation (2a) (with a stress tensor modulated by the stiffness degradation function) and not the static equilibrium corresponding to the minimality of the total potential energy. As will be shown through numerical examples, it has a direct impact on the apparent crack evolution law.

Equations (2a) and (2b) are the governing laws of the so-called phase-field models $[14,15,16]$ with a particular choice of damage constitutive laws (and a non-essential scaling of internal length $\ell \mapsto 2 \tilde{\ell})$

$$
a(\alpha)=(1-\alpha)^{2}, \quad w(\alpha)=w_{1} \alpha^{2}
$$

with $w_{1}=G_{\mathrm{c}} /(2 \ell)$. The physical properties of general gradient damage models have been carefully studied in $[18,19,2,20,21]$ in a quasi-static setting but most of those are still applicable here. In particular, the choice (3) leads to the absence of a purely elastic domain in which damage is zero and an elastic behavior $\sigma=A \varepsilon$ is observed. As is already pointed out in $[14,16]$, the stress is increasing (hardening) in the damage interval $\left(0, \frac{1}{4}\right)$ during a homogeneous 1-d traction test, which complicates the physical interpretation of the damage variable. Here in this paper, we will be focused on the following constitutive functions

$$
a(\alpha)=(1-\alpha)^{2}, \quad w(\alpha)=w_{1} \alpha
$$

with $w_{1}=3 G_{\mathrm{c}} /(8 \ell)$. The main advantage of this model (4) is the presence of a purely elastic domain controlled by a critical stress $\sigma_{\mathrm{c}}=\sqrt{w_{1} E}$, while the surface energy is still quadratic with respect to the damage variable leading to a minimal computational cost because of a constant Hessian matrix, see [2] for a comparison of these two models among others. 


\section{Numerical implementation}

The implementation of the space-time continuous model (1) is mainly adapted from [2, 22] for discretization schemes and numerical treatment of the damage equation (2b). The fields $\mathbf{u}$ et $\alpha$ are discretized in space by isoparametric finite elements with the same interpolation functions based on a mesh $\Omega_{h} \subset \Omega$ the typical size of which should be sufficiently small compared to the internal length $\ell$ in order to estimate correctly the surface energy $\mathcal{S}\left(\alpha_{t}\right)$. In explicit dynamics linear elements are largely preferred because of its lower computational cost and a simply obtainable diagonal lumped mass matrix.

The central difference Newmark scheme with $\beta=0$ is used for temporel discretization of the wave equation (2a), given its precision, its symplectic nature producing little numerical dissipation and its explicit character requiring no inversion of matrices at every time step. The conditional stability $\Delta t<\Delta t_{\mathrm{CFL}} \approx h / c$ is not very inconvenient in our applications as cracks can propagate at a speed comparable to the material speed of sound.

In absence of the temporel derivative of the damage field $\dot{\alpha}$, the energy minimization problem (2b) isn't a genuine evolution problem except that the irreversibility condition should be discretized conforming to the time steps. We obtain hence a bound-constrained convex minimization problem (or even a quadratic programming problem using constitutive laws (4)). which will be solved at the structural scale by the Gradient Projection (identification of active bounds) combined with the Conjugate Gradient method (approximated solution corresponding to the free variables), cf. [23].

In the time-continuous model the dynamic equilibrium (2a) and the damage stability criterion (2b) are coupled in the variational inequality (1). It turns out that our choice of the temporal discretization (explicit Newmark scheme) decouples automatically at every time step two separate and independant sub-problems respectively at $\mathbf{u}$ fixed and at $\alpha$ fixed. When using other implicit schemes, staggered or operator-split schemes should be used $[22,15]$. Combing spatial and temporal discretization, we obtain the following numerical model.

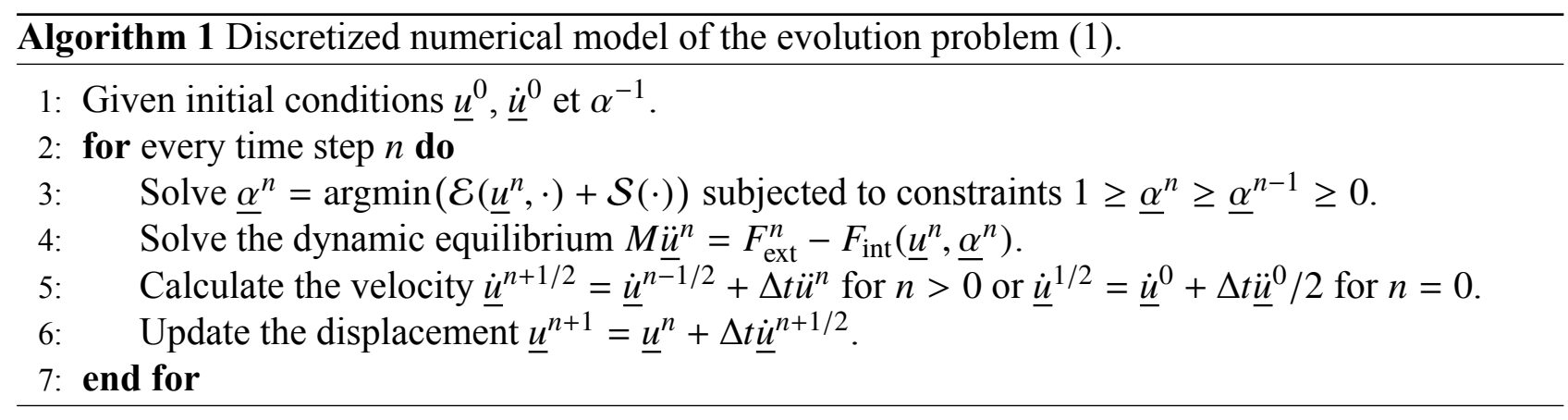

It is shown in [24] that the time-discrete model using an implicit Euler scheme [22] converges to the continuous one governed by the three principles when $\Delta t \rightarrow 0$. Our experience suggests the same using the explicit central difference scheme. Note that in the discrete model we make use only of the variational inequality (1). The energy balance criterion in the continuous model will be automatically satisfied when the time increment tends to zero.

\section{Links with the Griffith theory of dynamic fracture}

In order to better understand the proposed dynamic gradient damage model using a regularized description of cracks, we consider a mode III (antiplane) dynamic crack propagation case in a two dimensional plate $(0, L) \times(-H, H)$. The loading velocity $k$ is varied and its influence on the crack speed is studied. With a minor modification of the elastic energy $\mathcal{E}(\mathbf{u}, \alpha)$ similar to [22], the crack is enforced to propagate along a straight predefined path. The aim is to provide through this academic example an intuitive interpretation of the variational inequality (1) in terms of fracture mechanics languages. 


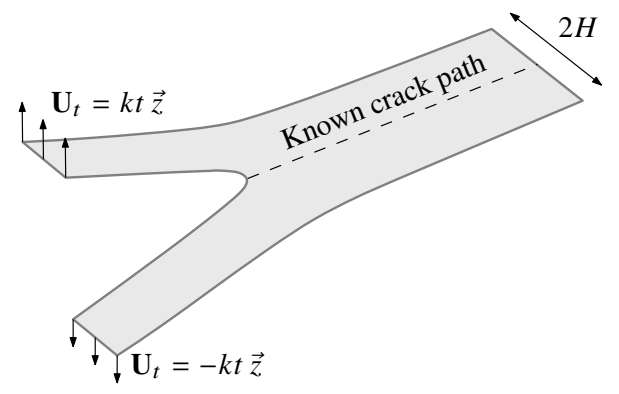

Figure 2: Mode III (antiplane) dynamic crack propagation case in a two dimensional plate $(0, L) \times$ $(-H, H)$ with a loading speed parametrized by $k$. A structured crossed triangular mesh with $h=0.01$ is used and the following parameters are adopted: $L=5, H=1, \rho=1, \mu=0.2, G_{\mathrm{c}}=0.01, \ell=0.05$.

Denoting the current crack length by $l(t)$, we have the approximation $\mathcal{S}(\alpha(t)) \approx\left(G_{\mathrm{c}}\right)_{\mathrm{eff}} l(t)$ with $\left(G_{\mathrm{c}}\right)_{\mathrm{eff}}=(1+3 h /(8 \ell)) G_{\mathrm{c}}$ the numerical amplified material toughness due to spatial discretization, see [7]. The crack speed can thus be obtained by linear regression during the steady propagation phase. This antiplane tearing example is physically similar to the 1-d film peeling problem studied using Griffith theory in [25] and the displacement field is well approximated by the 1-d result when the plate width $H$ is small. According to [25], the crack speed as a function of the loading velocity $k$ is given by

$$
\frac{\mathrm{d} l}{\mathrm{~d} U}(k)=\sqrt{\frac{\mu H}{G_{\mathrm{c}}+\rho H k^{2}}} \quad \text { or } \quad \frac{\mathrm{d} l}{\mathrm{~d} t}(k)=\sqrt{\frac{\mu H k^{2}}{G_{\mathrm{c}}+\rho H k^{2}}}
$$

from which we retrieve the quasi-static limit $\mathrm{d} l / \mathrm{d} U(0)=\sqrt{\mu H / G_{\mathrm{c}}}$ announced in [7] and the dynamic shearing velocity $\mathrm{d} l / \mathrm{d} t(\infty)=\sqrt{\mu / \rho}$, classical result of the Griffith theory of dynamic fracture [26].

In Fig. 3 (first two figures), we compare the numerical results with this 1-d analytical solutions and a very good agreement is found between them. In this particular case where the crack path is enforced, the crack advances according to the dynamic Griffith criterion $G(i)=\left(G_{\mathrm{c}}\right)_{\mathrm{eff}}$ during the steady propagation phase, as is shown in Fig. 3 (right). The (apparent) dynamic energy release rate is calculated using domain perturbation techniques [27] adapted in our gradient damage model case and is given by

$G_{t}=\int_{\Omega \backslash \Gamma_{t}} \boldsymbol{\sigma}\left(\mathbf{u}_{t}, \alpha_{t}\right) \cdot\left(\nabla \mathbf{u}_{t} \nabla \boldsymbol{\theta}_{t}\right)+\frac{1}{2} \rho \dot{\mathbf{u}}_{t} \cdot \dot{\mathbf{u}}_{t} \operatorname{div} \boldsymbol{\theta}-\frac{1}{2} \boldsymbol{\sigma}\left(\mathbf{u}_{t}, \alpha_{t}\right) \cdot \boldsymbol{\varepsilon}\left(\mathbf{u}_{t}\right) \operatorname{div} \boldsymbol{\theta}_{t}+\rho \ddot{\mathbf{u}}_{t} \cdot \nabla \mathbf{u}_{t} \boldsymbol{\theta}_{t}+\rho \dot{\mathbf{u}}_{t} \cdot \nabla \dot{\mathbf{u}}_{t} \boldsymbol{\theta}_{t}$

with $\boldsymbol{\sigma}\left(\mathbf{u}_{t}, \alpha_{t}\right)=a\left(\alpha_{t}\right) \mathrm{A} \boldsymbol{\varepsilon}\left(\mathbf{u}_{t}\right)$ the stress tensor and $\boldsymbol{\theta}_{t}$ a domain perturbation simulating a virtual crack extension at the current crack tip at time $t$.
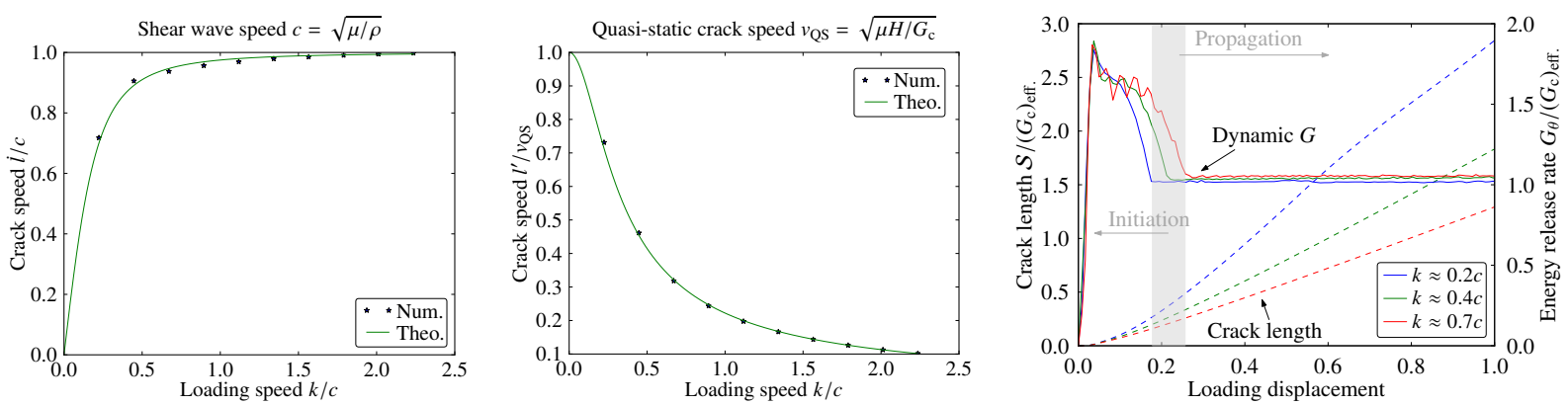

Figure 3: Crack speeds (with respect to time and imposed displacement) as a function of loading velocities: comparaison with the 1-d analytical solution using Griffith criterion $G(i)=\left(G_{\mathrm{c}}\right)_{\mathrm{eff}}$; Evolution of the calculated dynamic energy release rate. 


\section{Quasi-static limits of the dynamic model}

According to [28], our dynamic gradient damage model (1) converges to the quasi-static model of [2] with the directional stability condition replaced by its first-order static equilibrium and damage criterion condition, supposing temporel regularity of the crack (as in the classical Griffith theory). We verify this result by imposing a small loading speed $k / c \approx 0.2 \%$ in the above antiplane tearing case. From Fig. 4 (left) we see that dynamic solution coincides well with the quasi-static solution, showing that $G_{\mathrm{dyn}} \approx G_{\text {stat }} \approx\left(G_{\mathrm{c}}\right)_{\text {eff }}$ during the propagation phase. Next we consider a heterogeneous plate as did in [25] with a toughness $G_{\mathrm{c}}$ change from $\Gamma_{1}$ to $\Gamma_{2}$ at $x=1$ and two cases are studied: hardening case $\Gamma_{1}<\Gamma_{2}$ and softening case $\Gamma_{1}>\Gamma_{2}$. In the hardening case as predicted by analytical results of [25] based on classical Griffith theory, the crack comes to an halt at $x=1$ before a restart when the energy release rate re-attain the second material toughness $\Gamma_{2}=2 \Gamma_{1}$. Both quasi-static and dynamic solutions give the same result, as no crack jump is observed. This is not the case anymore when the material toughness $G_{\mathrm{c}}=\Gamma_{1}$ suddenly drops to a smaller value $\Gamma_{2}=\frac{1}{2} \Gamma_{1}$. In Fig. 4 (right), the quasi-static solution of [2] underestimates the crack jump and predicts no crack arrest, by relating directly the static energy release rate $G$ to the material toughness $\Gamma_{2}$ just after the toughness change. However, the correct way, as indicated by our dynamic solutions, is to satisfy the (quasi-static) energy conservation condition during the jump as analyzed by a complete dynamic calculation [25].
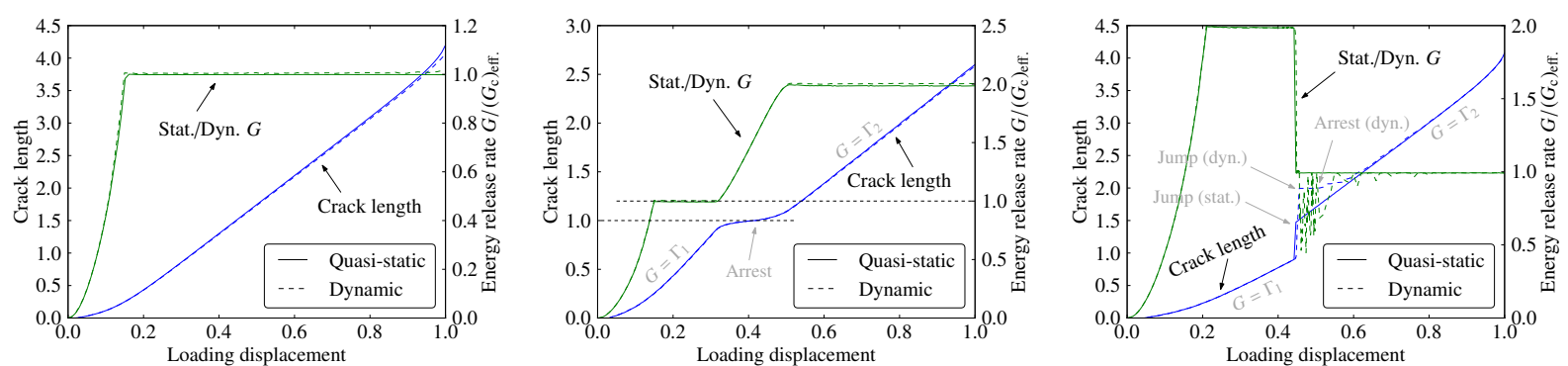

Figure 4: Crack lengths and calculated energy release rates at a very slow loading speed, in a homogeneous $G_{\mathrm{c}}$ plate (left) and a heterogeneous $G_{\mathrm{c}}$ plate (middle: hardening case $\Gamma_{1}<\Gamma_{2}$ and right: softening case $\Gamma_{1}>\Gamma_{2}$ ).

\section{Conclusions and outlook}

In this paper we have proposed a general class of dynamic gradient damage models as a natural extension of the original quasi-static one [1]. Our formulation contains the so-called phase-field models $[14,15,16]$ with a particular choice of damage constitutive laws (3). Through an academic antiplane tearing test with a predefined crack path, it is shown that this model with a regularized description of cracks is in line with the Griffith theory of dynamic fracture $G(i)=G_{\mathrm{c}}$ in this simplest case. However all the power of the model lies in the prediction of crack kinking or branching solely using the crack minimality criterion (2b), see Fig. 5 the result of which are in good accordance with experimental results [29]. Future work will be devoted to a better understanding of the kinking or branching mechanism predicted by this model.

\section{References}

[1] Kim Pham and Jean-Jacques Marigo. Approche variationnelle de l'endommagement : II. Les modèles à gradient. Comptes Rendus Mécanique, 338(4):199-206, 2010. 

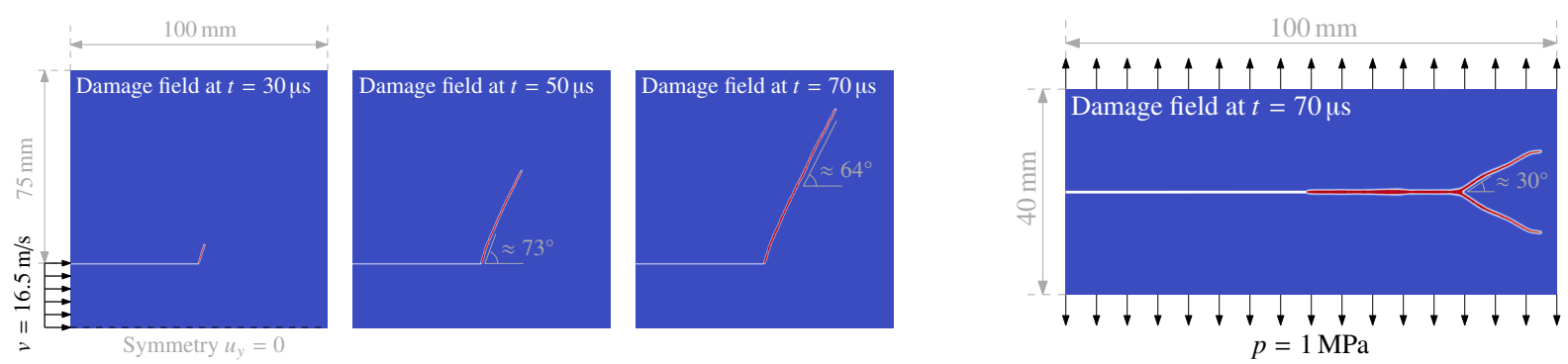

Figure 5: In-plane kinking and branching.

[2] Kim Pham, Hanen Amor, Jean-Jacques Marigo, and Corrado Maurini. Gradient damage models and their use to approximate brittle fracture. International Journal of Damage Mechanics, 20(4):618-652, 2011.

[3] Eric Lorentz and V. Godard. Gradient damage models: Toward full-scale computations. Computer Methods in Applied Mechanics and Engineering, 200(21-22):1927-1944, 2011.

[4] A. Benallal, R. Billardon, and G. Geymonat. Bifurcation and Localization in Rate-Independent Materials. Some General Considerations. In Q.S. Nguyen, editor, Bifurcation and Stability of Dissipative Systems, volume 327 of International Centre for Mechanical Sciences, pages 1-44. Springer Vienna, 1993.

[5] Ahmed Benallal and Jean-Jacques Marigo. Bifurcation and stability issues in gradient theories with softening. Modelling and Simulation in Materials Science and Engineering, 15(1):S283, 2007.

[6] Eric Lorentz and S. Andrieux. Analysis of non-local models through energetic formulations. International Journal of Solids and Structures, 40(12):2905-2936, 2003.

[7] Blaise Bourdin, Gilles A. Francfort, and Jean-Jacques Marigo. The Variational Approach to Fracture. Journal of Elasticity, 91(1-3):5-148, 2008.

[8] Paul Sicsic and Jean-Jacques Marigo. From gradient damage laws to Griffith's theory of crack propagation. Journal of Elasticity, 113(1):55-74, 2013.

[9] Paul Sicsic, Jean-Jacques Marigo, and Corrado Maurini. Initiation of a periodic array of cracks in the thermal shock problem: A gradient damage modeling. Journal of the Mechanics and Physics of Solids, 63:256-284, 2014.

[10] Blaise Bourdin, Jean-Jacques Marigo, Corrado Maurini, and Paul Sicsic. Morphogenesis and propagation of complex cracks induced by thermal shocks. Physical Review Letters, 112(1):014301, 2014.

[11] A. Mesgarnejad, B. Bourdin, and M.M. Khonsari. A variational approach to the fracture of brittle thin films subject to out-of-plane loading. Journal of the Mechanics and Physics of Solids, 61(11):2360-2379, 2013.

[12] A.A. León Baldelli, J.-F. Babadjian, B. Bourdin, D. Henao, and C. Maurini. A variational model for fracture and debonding of thin films under in-plane loadings. Journal of the Mechanics and Physics of Solids, 70:320-348, 2014.

[13] Gilles A. Francfort and Jean-Jacques Marigo. Revisiting brittle fracture as an energy minimization problem. Journal of the Mechanics and Physics of Solids, 46(8):1319-1342, 1998. 
[14] Michael J. Borden, Clemens V. Verhoosel, Michael A. Scott, Thomas J.R. Hughes, and Chad M. Landis. A phase-field description of dynamic brittle fracture. Computer Methods in Applied Mechanics and Engineering, 217-220(0):77-95, 2012.

[15] Martina Hofacker and Christian Miehe. Continuum phase field modeling of dynamic fracture: variational principles and staggered FE implementation. International Journal of Fracture, 178(1-2):113-129, 2012.

[16] Alexander Schlüter, Adrian Willenbücher, Charlotte Kuhn, and Ralf Müller. Phase field approximation of dynamic brittle fracture. Computational Mechanics, 54(5):1141-1161, 2014.

[17] Gianpietro Del Piero, Giovanni Lancioni, and Riccardo March. A variational model for fracture mechanics: Numerical experiments. Journal of the Mechanics and Physics of Solids, 55(12):2513-2537, 2007.

[18] Kim Pham and Jean-Jacques Marigo. Damage localization and rupture with gradient damage models. Frattura ed Integrità Strutturale, 19:5-19, 2012.

[19] Kim Pham and Jean-Jacques Marigo. From the onset of damage to rupture: construction of responses with damage localization for a general class of gradient damage models. Continuum Mechanics and Thermodynamics, 25(2-4):147-171, 2013.

[20] Kim Pham and Jean-Jacques Marigo. Stability of homogeneous states with gradient damage models: size effects and shape effects in the three-dimensional setting. Journal of Elasticity, 110(1):63-93, 2013.

[21] Kim Pham, Jean-Jacques Marigo, and Corrado Maurini. The issues of the uniqueness and the stability of the homogeneous response in uniaxial tests with gradient damage models. Journal of the Mechanics and Physics of Solids, 59(6):1163-1190, 2011.

[22] B. Bourdin, C. J. Larsen, and C. L. Richardson. A time-discrete model for dynamic fracture based on crack regularization. International Journal of Fracture, 168(2):133-143, 2011.

[23] J. Moré and G. Toraldo. On the Solution of Large Quadratic Programming Problems with Bound Constraints. SIAM Journal on Optimization, 1(1):93-113, 1991.

[24] Christopher J. Larsen, Christoph Ortner, and Endre Süli. Existence of Solutions to a Regularized Model of Dynamic Fracture. Mathematical Models and Methods in Applied Sciences, 20(7):1021-1048, 2010.

[25] P.-E. Dumouchel, J.-J. Marigo, and M. Charlotte. Dynamic fracture: an example of convergence towards a discontinuous quasistatic solution. Continuum Mechanics and Thermodynamics, 20(1):1-19, 2008.

[26] L. B. Freund. Dynamic Fracture Mechanics. Cambridge University Press, 1990.

[27] P. Destuynder and M. Djaoua. Sur une interprétation mathématique de l'intégrale de Rice en théorie de la rupture fragile. Mathematical Methods in the Applied Sciences, 3(1):70-87, 1981.

[28] Henrique Versieux. A relation between a dynamic fracture model and quasi-static evolution. ESAIM: M2AN, 2015.

[29] Jeong-Hoon Song, Hongwu Wang, and Ted Belytschko. A comparative study on finite element methods for dynamic fracture. Computational Mechanics, 42(2):239-250, 2008. 